\title{
Manipulating the voltage drop in graphene nanojunctions using a gate potential
}

\author{
Papior, Nick Rübner; Gunst, Tue; Stradi, Daniele; Brandbyge, Mads
}

Published in:

Physical Chemistry Chemical Physics

Publication date:

2016

Document Version

Publisher's PDF, also known as Version of record

Link back to DTU Orbit

Citation (APA):

Papior, N. R., Gunst, T., Stradi, D., \& Brandbyge, M. (2016). Manipulating the voltage drop in graphene nanojunctions using a gate potential. Physical Chemistry Chemical Physics.

\section{General rights}

Copyright and moral rights for the publications made accessible in the public portal are retained by the authors and/or other copyright owners and it is a condition of accessing publications that users recognise and abide by the legal requirements associated with these rights.

- Users may download and print one copy of any publication from the public portal for the purpose of private study or research.

- You may not further distribute the material or use it for any profit-making activity or commercial gain

- You may freely distribute the URL identifying the publication in the public portal

If you believe that this document breaches copyright please contact us providing details, and we will remove access to the work immediately and investigate your claim 


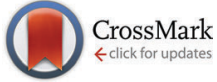

Cite this: DOI: $10.1039 / \mathrm{c5cp04613k}$

\title{
Manipulating the voltage drop in graphene nanojunctions using a gate potential $\dagger$
}

\author{
Nick Papior, ${ }^{\star a b}$ Tue Gunst, $^{\mathrm{ab}}$ Daniele Stradi ${ }^{\mathrm{ab}}$ and Mads Brandbyge $\mathrm{ab}^{\mathrm{ab}}$
}

Graphene is an attractive electrode material to contact nanostructures down to the molecular scale since it can be gated electrostatically. Gating can be used to control the doping and the energy level alignment in the nanojunction, thereby influencing its conductance. Here we investigate the impact of electrostatic gating in nanojunctions between graphene electrodes operating at finite bias. Using quantum transport simulations based on density functional theory, we show that the voltage drop across symmetric junctions changes dramatically and controllably in gated systems compared to non-gated junctions. In particular, for $\mathrm{p}$-type(n-type) carriers the voltage drop is located close to the electrode with positive(negative) polarity, the potential of the junction is pinned to the negative(positive) electrode. We trace this behaviour back to the vanishing density of states of graphene in the proximity of the Dirac point. Due to the electrostatic gating, each electrode exposes different density of states in the bias window between the two different electrode Fermi energies, thereby leading to a non-symmetry in the voltage drop across the device. This selective pinning is found to be independent of device length when carriers are induced either by the gate or dopant atoms, indicating a general effect for electronic circuitry based on graphene electrodes. We envision this could be used to control the spatial distribution of Joule heating in graphene nanostructures, and possibly the chemical reaction rate around high potential gradients.

www.rsc.org/pccp

\section{Introduction}

Graphene (Gr) shows great promise as a central material for future two-dimensional (2D) nanoelectronic applications. ${ }^{1,2}$ In particular, its semi-metallic character and its record high mean-free path ${ }^{3}$ makes it a top candidate for ultra-fast and flexible electronic components. ${ }^{4,5}$ Fuelled by these perspectives, nanostructured devices down to the molecular scale using electrodes based on Gr have recently been put forward. ${ }^{6-9}$ In their most generic form, these devices are composed by a $\mathrm{Gr}$ constriction where the narrowest junction consists of a $\mathrm{Gr}$ nanoribbon $(\mathrm{GNR})^{10,11}$ or an organic molecule. ${ }^{6-9}$ More complex structures such as Gr antidot lattices ${ }^{12,13}$ can also be viewed as consisting of a network of constrictions.

A unique feature of $\mathrm{Gr}$ electrodes is that their electronic properties can easily be tuned by electrostatic gating. In fact, electrostatic gates can be used to increase the carrier density in Gr up to above $10^{13} \mathrm{~cm}^{-2}$. ${ }^{14}$ For ion gating it has even been possible to reach carrier densities of $10^{14} \mathrm{~cm}^{-2}$ which correspond to a Fermi energy shift of about $1 \mathrm{eV} \cdot{ }^{15}$ It has been shown that

\footnotetext{
${ }^{a}$ Department of Nanotech, Technical University of Denmark, H.C. Ørsteds plads 345b, Kgs. Lyngby, Denmark. E-mail: nickpapior@gmail.com

${ }^{b}$ Center for Nanostructured Graphene (CNG), Denmark

$\dagger$ Electronic supplementary information (ESI) available. See DOI: 10.1039/c5cp04613k
}

gating can be used to tune the resonances localized in the narrowest part of the junction, ${ }^{16,17}$ as the electronic states of the electrodes are usually affected only weakly by the gate-induced capacitive field due to effective screening by the high density of states (DOS). ${ }^{18}$ However, for Gr electrodes, the lower DOS and its flat geometry, makes it comparable to the junction itself, and is thus likely to be perturbed similarly by gating. This peculiarity leads to a novel, yet largely unexplored, paradigm for graphenebased electronics, as the transport characteristics of the device ultimately depend on the response of the entire system to the gate. In electronic transport simulations, the effect of electrostatic gating and induced doping charge in the device has often been mimicked by rigidly shifting the position the Fermi-level/chemical potential in calculations without explicitly including the gate or dopants. ${ }^{19-22}$ However, despite accounting for some of the effects, these approaches completely neglect the self-consistent response of the device to the additional charge doping or the gate-induced electric field.

Here, we investigate these issues by extending the TranSIESTA electronic transport package, based on density functional theory and nonequilibrium Green function (DFT-NEGF), ${ }^{23,24}$ with the inclusion of a physically motivated gate model, see Fig. 1a. This improvement allows us to consider on an equal footing the effect of charge doping, capacitive gate field, and of the finite bias voltage in our DFT + NEGF simulations (see the Methods section 


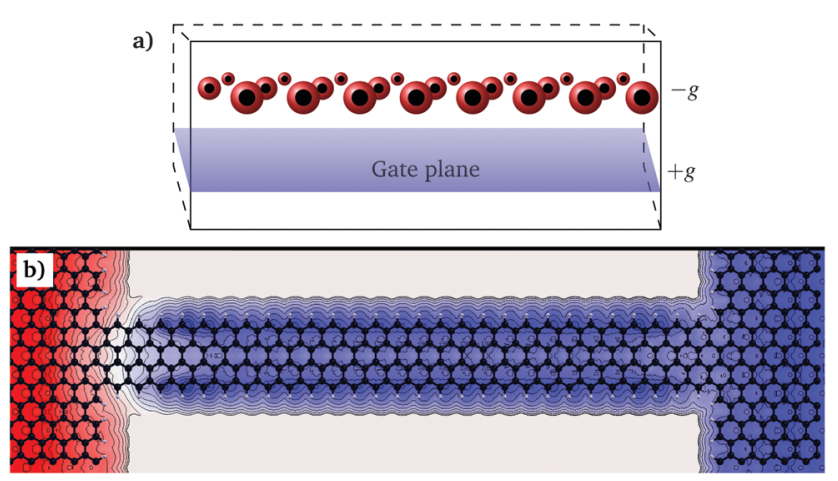

Fig. 1 (a) implementation of the field effect gate model. Redistribution of charge from atoms to gate plane. (b) resulting voltage drop for a $8.3 \mathrm{~nm}$ long constriction including a field effect gate of $n=2 \times 10^{13} \mathrm{e}^{-} \mathrm{cm}^{-2}$ and a bias of $0.5 \mathrm{~V}$. Contour lines are separated by $0.022 \mathrm{~V}$.

for additional details of the implementation). We apply this methodology to $\mathrm{Gr}$ constrictions consisting of nanoribbon junctions between Gr electrodes. For these we demonstrate how the transport characteristics depend in a non-trivial way on the applied source/drain and gate voltages. As seen in Fig. $1 \mathrm{~b}$, upon gating and bias, the voltage drop is pinned to the electrode of a given polarity depending on the doping type and the bias, even for a constriction of $8.3 \mathrm{~nm}$. We can relate the phenomenon to the gate-dependent behavior of the voltage drop in the system which, in turn, can be traced back to the energy dependence of the DOS in the $\mathrm{Gr}$ electrodes. The electronic structure of the semi-metallic Gr electrodes displays zero DOS at its charge neutrality point, and a linear increase of the DOS away from it (V-shape). Our analysis demonstrates how the V-shaped DOS in the electrodes control the voltage drop in the Gr junctions indicating a quite generic scenario.

The control of the position of the voltage drop on the nanoscale with gate could be useful in practical applications. We envision this feature, f.ex., could be used to tune the spatial distribution of Joule heating in the device and influence its breaking at the nanoscale. ${ }^{21,25}$ Our results highlight the importance of using fully self-consistent electronic transport simulations to predict and design the gating behavior under operating conditions of the emerging class of devices with electrodes having a vanishing DOS. ${ }^{18}$

\section{Results and discussion}

We have applied our method to two geometrically similar, "leftright" symmetric Gr nanojunctions, formed by a Gr nanoribbon connected to pristine Gr electrodes, see Fig. 2 and 3. For zero gate/doping $(g=0)$ the former yield an electron-hole symmetric electronic structure (hydrogen GNR), whereas the latter yield a e-h non-symmetric electronic structure (Oxygen GNR) ${ }^{26-28}$ The hydrogen-terminated system is also investigated using dopant atoms instead of the electrostatic gating. ${ }^{11}$ The simulation unit cell has an area of $\sim 200 \mathrm{Gr}$ unit cells. The gate is placed 20 beneath the planar Gr structure and we sweep the gating levels $(g)$ according to $g \times 10^{13} \mathrm{e}^{-} \mathrm{cm}^{-2}$.

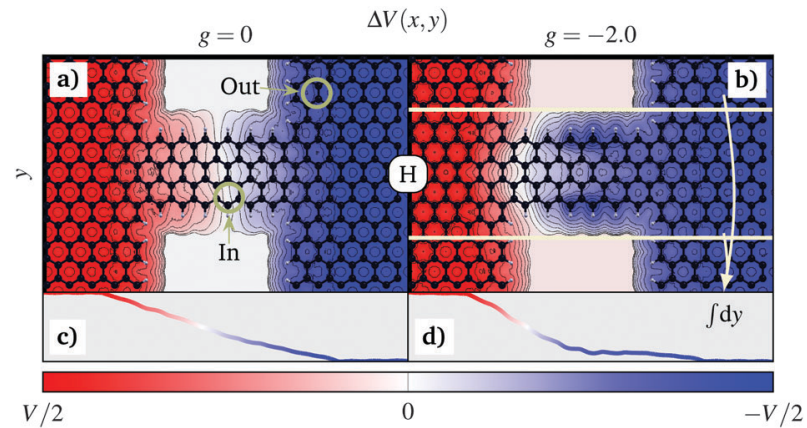

Fig. 2 Electronic Hartree potential drop integrated perpendicular to the plane and above a cutoff electron density $\rho_{\varepsilon}=0.008 \mathrm{e} \AA^{-3}$ and projected to the graphene plane for the Hydrogen GNR, (a) and (b). (c) and (d) are the contour plot further integrated in the box indicated in (b). The non-gated system shows a linear gradient, whereas for $g<0$ ( $n$-doped) a pinning of the potential towards the right (positive) electrode.

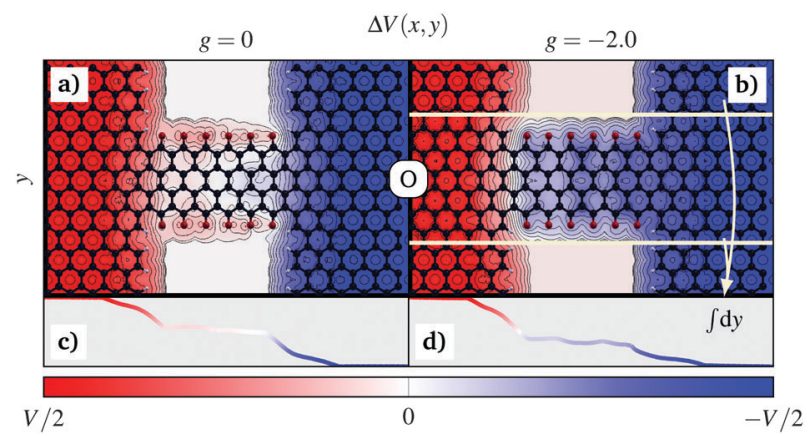

Fig. 3 Electronic Hartree potential drop integrated perpendicular to the plane and above a cutoff electron density $\rho_{\varepsilon}=0.008$ e $\AA^{-3}$ and projected to the graphene plane for the Oxygen GNR, (a) and (b). (c) and (d) are the contour plot further integrated in the box indicated in (b). The non-gated system shows a gradient at the GNR boundary, whereas for $g<0$ ( $n$-doped) a pinning of the potential towards the right (positive) electrode.

In Fig. 2a and $\mathrm{b}$, we plot the potential drop across the $\mathrm{Gr}$ constriction at $0.5 \mathrm{~V}$ for $g=0$, (a), and n-doped with $g=-2$ gating, (b). The potential profile has been integrated in the perpendicular direction to the $\mathrm{Gr}$ surface for electronic densities above $\rho_{\varepsilon}=0.008$ e $\AA^{-3}$ projected onto the $x-y$ plane. The lower panels, (c) and (d), is a further projection onto the transport direction $(x)$ as indicated in Fig. 2b. At $g=0$ we obtain an anti-symmetric potential drop in the transport direction $(\Delta V(x)=-\Delta V(-x))$ as expected for a fully e-h and left-right symmetric constriction. On the other hand, in the $g=-2$ (n-doped Gr), we see a clear pinning of the potential profile to the positive electrode, the potential drop at the negative electrode. Conversely, calculations with $g=+2$ (p-doping) with $0.5 \mathrm{~V}$ display a pinning at the negative electrode, while for $g=+2$ and $-0.5 \mathrm{~V}$ we regain the plot shown. This confirms the geometric symmetry.

In Fig. 4 we show the electron transmission spectra for the hydrogen passivated constriction at $0 \mathrm{~V}$, (a), and $0.5 \mathrm{~V}$, (b), for different values of $g$ each vertically shifted 1/2. As a measure of gating we track the position of two resonances, $\square$ and $O$ dots, corresponding to a resonance in the constriction located at the 
a)

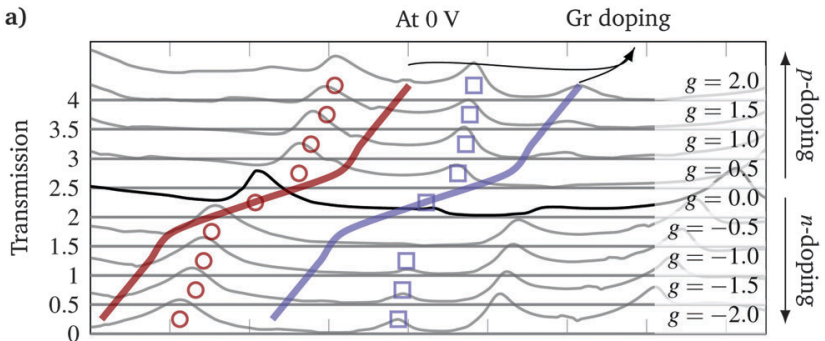

b)

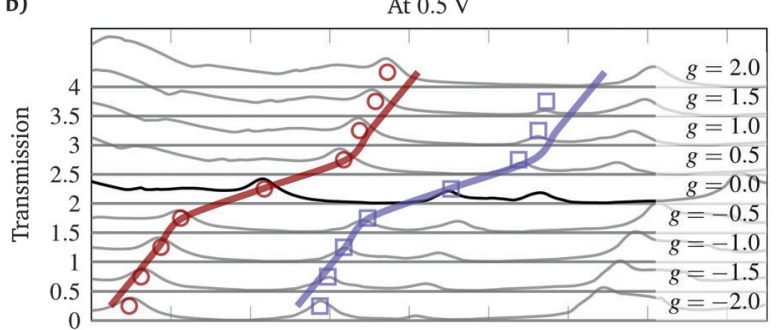

c)

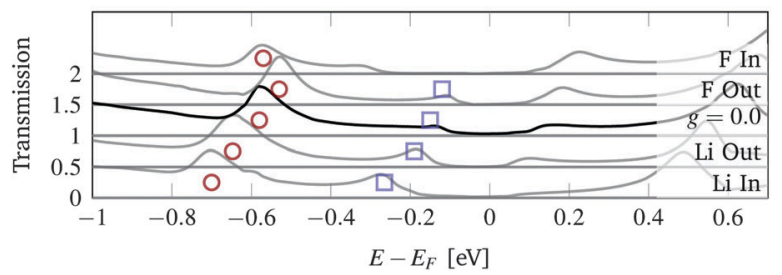

Fig. 4 Transmission spectra for the constriction at various doping levels for $0 \mathrm{~V},(\mathrm{a}), 0.5 \mathrm{~V},(\mathrm{~b})$, and for dopants, (c). The middle line (black) at zero gating is a symmetric transmission function with two distinct resonances (marked $\square$, and 0 ). Gating the constriction shifts the resonances as indicated by the displacements of the marks. The full lines, crossing vertically the different doping levels, indicates the graphene electrode Fermi level shift due to the doping aligned at the $g=0$ mark.

edge and in the center, respectively. The middle thick line is the transmission for $g=0$, and is equivalent to earlier results where these resonances are discussed. ${ }^{21}$ In addition, we plot the energy shift of the Dirac point for pristine graphene as vertical lines aligned at each of the two resonances at $g=0$. These vertical lines match exactly the shift in chemical potential due to the doping in the electrodes. Discrepancies between the electrode gating (lines) and the resonance positions (dots) illustrate the difference in just rigidly shifting the resonances according to electrode doping, and a fully self-consistent calculation of the resonance positions. Importantly, at $0 \mathrm{~V}$ we find that the resonance peaks does not simply follow the gating. Moreover, the two peaks are shifting/gated independently of each other; the center resonance peak, $\mathrm{O}$, follows the pristine doping closer than the edge resonance peak, $\square$, due to a difference in electrode coupling between the resonances. On the other hand, at $0.5 \mathrm{~V}$ we find that both peaks follow the pristine graphene electrode doping. As shown in Fig. 2b, the junction behaves as an extension of the positive electrode and therefore the resonance position is pinned at the Fermi level of this particular electrode. The selfconsistent calculation is needed to capture the correct transition with bias from semi-independent resonances to the pinned behavior. The same calculation was performed on a $8.3 \mathrm{~nm}$ long ribbon Fig. $1 \mathrm{~b}$ exhibiting the same pinning feature.
Fig. 3 are for the Oxygen terminated graphene nanoribbon. This nanoribbon has no e-h electronic DOS symmetry. ${ }^{26-28}$ Similarly to the hydrogen system we calculate for $g=0$ and $g=-2$ at $0.5 \mathrm{~V}$. a shows that the Oxygen edges pins slightly to the negative electrode for zero gating, while gating, (b), the entire ribbon is pinned to the positive electrode, equivalent to the hydrogen case Fig. $2 \mathrm{~b}$. This is also seen in the projected potential profiles Fig. $3 \mathrm{c}$ and d. This confirms that the selectivity of the potential profile in the gated devices does not rely on the e-h symmetry of the junction, and conjectures the generality of this behavior in systems with electrodes having V-shaped DOS around $E_{\mathrm{F}}$, regardless of the electronic structure of the central part connecting the two electrodes.

The generic behavior of the potential drop just outlined is summarized in Fig. 5, which shows the one-dimensional potential drop calculated for the hydrogen-terminated constriction for a number of different gates and positive bias voltages, similar to that of Fig. $2 \mathrm{c}$ and d. Independently on the particular value of the bias voltage applied, gating the system always leads to a marked asymmetry of the potential drop across the constriction. For any value of n-doping, the potential drop pins always to the positive (right) electrode for positive bias. Similarly, for any value of p-doping, the system couples to the negative (left) electrode for positive bias. These results further demonstrate the general phenomenon that does not depend on the particular values of applied gate or bias voltage. Furthermore, our calculations highlight the important fact that the charge neutrality point for the electrodes is a special case which does not extrapolate to the gated case. This becomes even more important if one considers the experimental difficulties in retaining a charge neutral sample. ${ }^{29,30}$

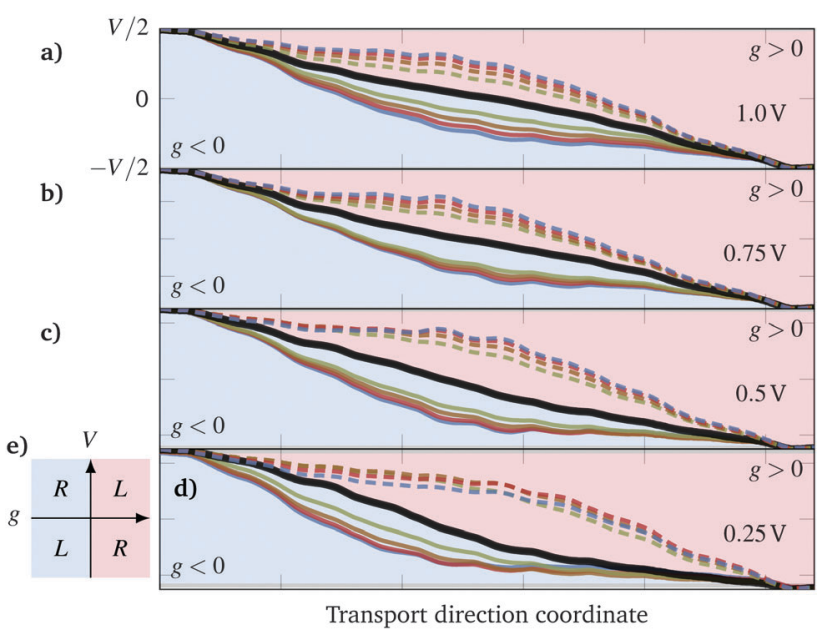

Fig. 5 (a)-(d) Integrated Hartree potential profile in a region of width corresponding to the ribbon along the entire constriction for varying gate levels and applied bias. The thick middle line is the potential profile for $g=0$. The blue regions correspond to $n$-doped graphene (full lines), while red are $p$-doped graphene (dashed lines). The non-gated calculations show a linear behavior whereas gated systems have a non-symmetry between the left and right electrode DOS breaking the left-right antisymmetry in the potential drop. (e) summarizes the trends where L/R means pinning to the left/right electrode. 


\section{Voltage drop model}

We will now consider a simple model which can explain the electrode selectivity of the voltage drop depending on the doping/electrostatic gating. Fig. 6 is a guided reference for the following discussion. The position of the voltage drop can be obtained by considering the change in charge in the scattering region when applying a bias. If the scattering region becomes more positive, one can view it as the positive electrode extending into the scattering region and thus the voltage drop will occur closer to the negative electrode and vice versa. The change in charge in the scattering region is linked to the change in injected charge from left and right electrodes in the bias window, as noted in the Methods section. The linear dependence of the DOS in the graphene electrodes makes the coupling/broadening functions of the scattering region display the energy dependence,

$$
\Gamma_{\mathrm{L} / \mathrm{R}}(E) \propto\left|E-\mu_{\mathrm{L} / \mathrm{R}}+E_{\mathrm{F}}\right|,
$$

where $E=0$ corresponds to the equilibrium Fermi level, $E_{\mathrm{F}}$ is the shift of Fermi level due to doping, $E_{\mathrm{F}} \propto \sqrt{g}$, and $\mu_{\mathrm{L} / \mathrm{R}}$ is the change in the chemical potential of left/right electrodes with applied voltage bias $(V)$. We will use $\mu_{\mathrm{L}}=e V / 2$ and $\mu_{\mathrm{R}}=-e V / 2$, and take $V>0$. This definition means that the scattering region as a starting point will not preferentially select the left or right electrode for an electron-hole symmetric system, and the potential drop profile will be spatially anti-symmetric, $\Delta V(x)=-\Delta V(-x)$. We will now consider the voltage bias as a "perturbation" onto the system without bias, and calculate the change in charge in the scattering region. Thus we first neglect the change in potential set up by the change in charge, which again will impact the charge in the self-consistency. With this we have the density of scattering states from left and right, $A_{\mathrm{L} / \mathrm{R}} \propto \Gamma_{\mathrm{L} / \mathrm{R}} \propto\left|E-\mu_{\mathrm{L} / \mathrm{R}}+E_{\mathrm{F}}\right|$, and the change in electrons(holes) injected from left(right) electrode can be written as,

$$
\begin{aligned}
\delta \mathrm{e} & =\int_{0}^{e V / 2} A_{\mathrm{L}}(E) \mathrm{d} E \propto \frac{e V}{2}\left(E_{\mathrm{F}}-\frac{e V}{4}\right), \\
\delta \mathrm{h} & =\int_{-e V / 2}^{0} A_{\mathrm{R}}(E) \mathrm{d} E \propto \frac{e V}{2}\left(E_{\mathrm{F}}+\frac{e V}{4}\right)
\end{aligned}
$$

where we assume $|V / 2|<\left|E_{\mathrm{F}}\right|$. The scenario is shown schematically in Fig. 6b showing more injection of positive carriers $\delta \mathrm{h}>\delta \mathrm{e}$. Thus the scattering region will as the first response to the
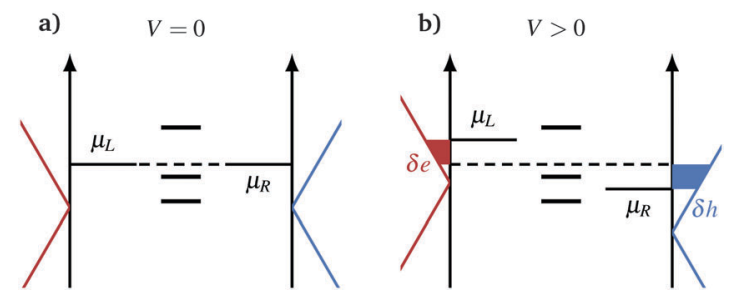

Fig. 6 Illustration of non-symmetric coupling induced by doping out of symmetry. (a) Shows the zero bias configuration with broken e-h symmetry due to doping, $g<0$. (b) Shows a difference among the electrode contributions in the bias window which pins the system to the right electrode.

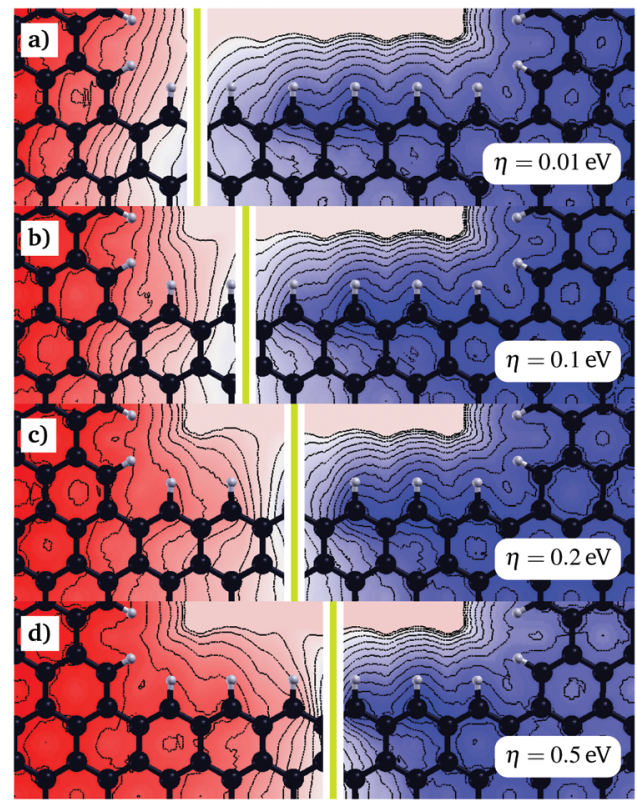

Fig. 7 Change of potential drop vs. level broadening parameter, $\eta_{\mathrm{L} / \mathrm{R}}$, for $0.5 \mathrm{~V}$. Increasing values smear out the electrode DOS which evens out the electronic contribution from both electrodes in the bias window. The voltage drop becomes anti-symmetric at even charge injection rates from the two electrodes (large smearing).

nonequilibrium filling become more positive and we conclude that for n-doping, $g<0$ and $E_{\mathrm{F}}>0$, the positive electrode will "extend" into the constriction resulting in a voltage drop at the negative electrode, as seen in Fig. 5. We stress that this behavior stems from the vanishing DOS of graphene at the Dirac point yielding a large relative difference between the electron/hole contributions. Contrary if we take $E_{\mathrm{F}}$ to be very large in eqn (2) and (3) we get $\delta \mathrm{e} \approx \delta \mathrm{h}$ and the constriction does not change its charge. Indeed, the pinning effect is smaller at $1 \mathrm{~V}$ compared to $0.5 \mathrm{~V}$ as seen in Fig. 5a vs. 5c. This is due to the DOS of one lead being very close to zero at $0.5 \mathrm{~V} ; \mu_{i}-E_{\mathrm{F}} \approx E_{\mathrm{D}}$ with $E_{\mathrm{D}}$ being the Dirac point, and hence a much larger relative difference in DOS.

In order to substantiate that the voltage drop is controlled by the vanishing electrode DOS we smear the DOS energy dependence gradually into a flat function by introducing an artificial increase in the broadening parameter, $\eta$, for the electrode self-energies in eqn $(4)$. Hence $\Gamma_{\mathrm{L}}(E) \approx \Gamma_{\mathrm{R}}(E)$ for $\eta \gg 0$ irrespective of the applied bias and gating. This forces $\delta \mathrm{e} \approx \delta \mathrm{h}$ and a resulting anti-symmetric voltage drop. Fig. 7 shows the voltage drop in the middle part of the constriction for four $\eta$ values. Clearly the anti-symmetric voltage drop is regained when $\eta_{\mathrm{L}, \mathrm{R}} \geq 0.5 \mathrm{eV}$. Note that since we have not made assumptions in the model about the nature of the constriction we anticipate that it can straightforwardly be applied to similar systems between graphene electrodes in the high-conductance regime.

\section{Constriction, hydrogen terminated with dopants}

Since Gr consists entirely of surface atoms it is also extraordinarily susceptible to external influences such as chemical modification or charged impurities. We will now discuss the influence of 
modifying the passivation or having adatoms ${ }^{31-34}$ as a source of charge doping alternative to the electrostatic gating. We examine the effect of a donating lithium ( $\mathrm{Li}$ ) or an accepting flourine (F) adatom placed either inside or outside the constriction at the positions shown in Fig. 2a. The Li or F atoms are positioned above the center of a hexagon, or ontop a Carbon atom, respectively. In Fig. 4c we show the transmission for the different adatom configurations. The transmission spectra indicate that very little scattering due to the dopants themselves takes place, especially when the adatoms are positioned outside the constriction. The doping effect is clearly seen from the shift in the two resonance peak positions. Li will n-dope the graphene constriction while F p-dope it. Surprisingly, we find that most of the charge transfer to the device resonances is maintained when the dopants are moved outside the constriction. This suggests that nanostructured graphene devices will not necessarily be very sensitive to the actual position of the adatoms. In the case of $\mathrm{F}$ it is actually more efficient outside the constriction. Comparing the most significant peak with the field effect gating transmission curves we find that Li donates at least 0.2 electrons while $\mathrm{F}$ accepts at least 0.3 electrons from graphene. In addition, we find that a pinning of the potential to the positive/negative electrode occurs for $\mathrm{Li}$ (n-doping)/F(p-doping) for positive bias, consistent with the potential drops obtained from field effect gating (see Fig. 2). Adatoms may therefore provide an alternative way to manipulate the voltage drop by pinning the potential to either of the two electrodes. This underlines the conclusion that the main effect is determined by the addition or removal of charge from the device, together with the uneven injection rates from the electrodes.

\section{Conclusion}

We have implemented an electrostatic gate method which introduce charge carriers and the corresponding electric field in a capacitor-like setup in self-consistent DFT-NEGF calculations with open boundary conditions to semi-infinite electrodes. The gate method has been applied to several graphene constrictions where the narrowest junction corresponds to a graphene nanoribbon with either hydrogen or oxygen passivation. For positive voltage bias and with electrostatic gating the junction potential gets preferentially pinned to the positive(negative) electrode for $\mathrm{n}(\mathrm{p})$-type doping charge, and vice versa for polarity changes of gating and/or bias. Thus the position of the voltage drop can be manipulated by the gate potential or correspondingly from charge doping from adatoms. The constrictions was found to couple selectively to the electrode with the highest DOS contribution in the bias window. The behavior was traced back to the vanishing DOS of graphene close to the Dirac point. A simple perturbation model showed how the selectivity is due to the low DOS of graphene around the Fermi level, irrespective of the details of the junction electronic structure. The V-shaped DOS is also true for the local DOS at armchair edges. ${ }^{19}$ Thus we anticipate that our results also apply to molecular junctions more weakly coupled via a barrier to armchair edges of graphene. We suggest that this selectivity and high potential gradient can be utilized in experiments on nanostructured graphene or similar 2D materials to control regions of reactivity, manipulate polar adsorbates, or providing control of and insights into the local Joule heating. ${ }^{25,35}$ We expect that Kelvin Atomic Force Microscopy, ${ }^{36}$ Scanning Tunnelling Potentiometry ${ }^{37}$ or Low-Energy Electron Potentiometry ${ }^{38}$ to be suitable experimental techniques to examine the effect pointed out here in nanostructured graphene.

\section{Methods}

The simulations have been performed using the SIESTA/TranSIESTA code with the PBE-GGA functional for exchange-correlation ${ }^{39}$ and a SZP basis-set. A confinement radii determined from an energy shift of $230 \mathrm{meV}$. The real-space grid cutoff was $230 \mathrm{Ry}$. The electronic temperature has been set to $25 \mathrm{meV}$ ( $50 \mathrm{meV}$ for the O-terminated constriction). Unless stated otherwise, the smearing parameter $\eta$ was set to $10^{-2} \mathrm{eV}$. The geometries were relaxed until all forces were smaller than $5 \times 10^{-2} \mathrm{eV} \AA^{-1}$. Five transverse $k$-points were used in the electronic structure calculation. This was increased to between 25 and $50 k$-points in the transport calculations. The transmission data have subsequently been interpolated. ${ }^{40}$ A vacuum gap of 120 was used in the direction normal to the constriction plane.

Our field effect setup consists of a gate electrode, a dielectric, and the system, here being the graphene nanojunctions. Applying a gate voltage charges the system and electrodes like in a capacitor setup, thus inducing an electrostatic potential gradient across the dielectric, which in this implementation is vacuum. The additional charge will redistribute to create a polarization in the system along the electric field direction. Such field effect setups can be realized in open-boundary DFT calculations by employing a nonequilibrium Green function (NEGF) scheme, ${ }^{24,41}$ or by solving the Poisson equation with appropriate boundary conditions. ${ }^{42,43}$ The former is a computationally expensive calculation compared to the latter.

Analogous to a plate capacitor setup we assume that an applied gate voltage induces an electron charge $-\delta e^{-}$in the system and a corresponding counter-charge $+\delta e^{-}$in the gate plane. This situation is accounted for by charging the system with a given electron charge $g=-\delta e^{-}$, and by distributing homogeneously the corresponding counter-charge $+\delta e^{-}$in a well defined region of the unit-cell, denoted gate, so that the overall system + gate remains charge neutral. The setup is shown schematically in Fig. 1a. Thus for $g>0$ we have a p-doped system, similarly for $g<0$ we have a n-doped system. Solving the Poisson equation inherently calculates the electric field between the gate and the system. As the calculation cell is periodic we apply the slab dipole correction ${ }^{42}$ to terminate the periodic electric field induced by the charge redistribution. The gating method can readily be adopted to transport calculations using NEGF if the gate is uniformly applied to the electrodes and the device. Additionally, the gate at the electrodes must have a resulting electric field perpendicular to the applied bias to assert the correct boundary conditions. Our implementation resembles that of Brumme ${ }^{44,45}$ except that we use a linear combination of atomic orbitals method, which means that 
the dielectric need not be simulated by a potential barrier to limit electronic penetration.

We note that the DFT-NEGF ${ }^{24}$ calculation relies on calculating the density by occupying the left and right scattering states to the different respective chemical potentials. This is done by integrating the left/right spectral density matrices, $\mathbf{A}_{\mathrm{L} / \mathrm{R}}$, given in terms of the retarded Greens function, $\mathbf{G}$,

$$
\begin{gathered}
\mathbf{A}_{\mathrm{L} / \mathrm{R}}(E)=\mathbf{G}(E) \Gamma_{\mathrm{L} / \mathrm{R}}(E) \mathbf{G}^{\dagger}(E), \\
\mathbf{G}(E)=\left[(E+i \eta) \mathbf{S}-\mathbf{H}-\Sigma_{\mathbf{L}}(E)-\Sigma_{\mathrm{R}}(E)\right]^{-1} .
\end{gathered}
$$

Here $\mathbf{H}, \mathbf{S}, \Gamma_{\mathrm{L} / \mathrm{R}}(E)=i\left[\Sigma_{\mathrm{L} / \mathrm{R}}(E)-\Sigma_{\mathrm{L} / \mathrm{R}}^{\dagger}(E)\right]$ are the Hamiltonian, the overlap and the electrode broadening matrices. The parameter $\eta \rightarrow 0^{+}$introduce a vanishingly small broadening of DOS. However, a finite $\eta$ broadens the electrode DOS.

The simple Voltage drop model is developed based on the following more detailed description. We consider a left-right symmetric conductor. In nonequilibrium the density (matrix) can formally be written at as an "equilibrium" contribution corresponding to the equilibrium Fermi energy, $E_{\mathrm{F}}$, plus two "nonequilibrium" contributions originating from the change in filling of left and right originating scattering states, say, $\mu_{\mathrm{L}}>E_{\mathrm{F}}>\mu_{\mathrm{R}}$. The "nonequilibrium" terms corresponding to negative charge injection from the negative electrode, and positive charge injection from the positive electrode,

$$
\rho=-\frac{1}{\pi} \int \mathrm{d} E \operatorname{Im}(E) n_{\mathrm{F}, E_{F}}+\delta \mathrm{e}-\delta \mathrm{h},
$$

where $\delta \mathrm{e}$ and $\delta \mathrm{h}$ are defined in eqn (2) and (3). We choose $E_{\mathrm{F}}=\left(\mu_{\mathrm{L}}+\mu_{\mathrm{R}}\right) / 2$ and consider the different fillings as a perturbation. If we neglect the resulting Landauer dipole field in $\mathbf{H}$, which appear in the response to this perturbation in the self-consistent DFT-NEGF calculation, then the first "equilibrium" term can not break left-right symmetry and result in a left-right symmetric density. It is then clear that the symmetry breaking and charge in the device is determined by the competition between the latter two contributions which are of opposite sign.

The systems studied here belong to the class highly conducting carbon junctions for which the DFT-NEGF method has been compared favorably to detailed experiments both in the linear ${ }^{46,47}$ and non-linear conductance regime. ${ }^{48}$ In any case, we are here mainly interested in the qualitative aspects of the behavior of the voltage drop.

\section{Acknowledgements}

We thank Osamu Sugino and Pablo Ordejón for useful comments on the method employed, and the Danish e-Infrastructure Cooperation (DeIC) for providing computer resources. The Center for Nanostructured Graphene (CNG) is sponsored by the Danish Research Foundation, Project DNRF58. DS acknowledges the support from the H. C. Ørsted COFUND postdoctoral program at DTU. The contour plots have been prepared with XCrySDen. ${ }^{49}$

\section{References}

1 A. Geim and K. S. Novoselov, Nat. Mater., 2007, 6(3), 183-191.

2 H. Raza, Graphene nanoelectronics: metrology, synthesis, properties and applications, Springer, 2011.

3 K. I. Bolotin, K. J. Sikes, Z. Jiang, M. Klima, G. Fudenberg, J. Hone, P. Kim and H. L. Stormer, Solid State Commun., 2008, 146, 351-355.

4 K. S. Kim, Y. Zhao, H. Jang, S. Y. Lee, J. M. Kim, K. S. Kim, J.-H. Ahn, P. Kim, J.-Y. Choi and B. H. Hong, Nature, 2009, 457, 706-710.

5 T. Georgiou, R. Jalil, B. D. Belle, L. Britnell, R. V. Gorbachev, S. V. Morozov, Y.-J. Kim, A. Gholinia, S. J. Haigh, O. Makarovsky, L. Eaves, L. A. Ponomarenko, A. K. Geim, K. S. Novoselov and A. Mishchenko, Nat. Nanotechnol., 2013, 8, 100-103.

6 Y. Cao, S. Dong, S. Liu, Z. Liu and X. Guo, Angew. Chem., Int. Ed., 2013, 52, 3906.

7 C. Jia, J. Wang, C. Yao, Y. Cao, Y. Zhong, Z. Liu, Z. Liu and X. Guo, Angew. Chem., Int. Ed., 2013, 52, 8666.

8 K. Ullmann, P. B. Coto, S. Leitherer, A. Molina-Ontoria, N. Martn, M. Thoss and H. B. Weber, Nano Lett., 2015, 15, 3512-3518.

9 H. Sadeghi, J. A. Mol, C. S. Lau, G. A. D. Briggs, J. Warner and C. J. Lambert, Proc. Natl. Acad. Sci. U. S. A., 2015, 112, 2658-2663. 10 S. Dutta and S. K. Pati, J. Mater. Chem., 2010, 20, 8207.

11 Q. Tang, Z. Zhou and Z. Chen, Nanoscale, 2013, 5, 4541.

12 T. G. Pedersen, C. Flindt, J. Pedersen, N. A. Mortensen, A. Jauho and K. Pedersen, Phys. Rev. Lett., 2008, 100, 136804.

13 J. Bai, X. Zhong, S. Jiang, Y. Huang and X. Duan, Nat. Nanotechnol., 2010, 5, 190-194.

14 J. Ye, M. F. Craciun, M. Koshino, S. Russo, S. Inoue, H. Yuan, H. Shimotani, A. F. Morpurgo and Y. Iwasa, Proc. Natl. Acad. Sci. U. S. A., 2011, 108, 13002-13006.

15 Y. Yin, Z. Cheng, L. Wang, K. Jin and W. Wang, Sci. Rep., 2014, 4, 5758.

16 H. Song, Y. Kim, Y. H. Jang, H. Jeong, M. A. Reed and T. Lee, Nature, 2009, 462, 1039-1043.

17 F. Prins, A. Barreiro, J. W. Ruitenberg, J. S. Seldenthuis, N. Aliaga-Alcalde, L. M. K. Vandersypen and H. S. J. van der Zant, Nano Lett., 2011, 11, 4607-4611.

18 E. J. G. Santos and E. Kaxiras, Nano Lett., 2013, 13, 898-902. 19 D. A. Ryndyk, J. Bundesmann, M.-H. Liu and K. Richter, Phys. Rev. B: Condens. Matter Mater. Phys., 2012, 86, 195425.

20 B. K. Nikolić, K. K. Saha, T. Markussen and K. S. Thygesen, J. Comput. Electron., 2012, 11, 78-92.

21 T. Gunst, J.-T. Lü, P. Hedegård and M. Brandbyge, Phys. Rev. B: Condens. Matter Mater. Phys., 2013, 88, 161401.

22 J.-T. Lü, R. B. Christensen, G. Foti, T. Frederiksen, T. Gunst and M. Brandbyge, Phys. Rev. B: Condens. Matter Mater. Phys., 2014, 89, 081405.

23 J. M. Soler, E. Artacho, J. D. Gale, A. Garca, J. Junquera, P. Ordejón and D. Sánchez-Portal, J. Phys.: Condens. Matter, 2002, 14, 2745-2779.

24 M. Brandbyge, J.-L. Mozos, P. Ordejón, J. Taylor and K. Stokbro, Phys. Rev. B: Condens. Matter Mater. Phys., 2002, 65, 1-17.

25 R. Murali, Y. Yang, K. Brenner, T. Beck and J. D. Meindl, Appl. Phys. Lett., 2009, 94, 243114. 
26 F. Cervantes-Sodi, G. Csányi, S. Piscanec and A. C. Ferrari, Phys. Rev. B: Condens. Matter Mater. Phys., 2008, 77, 165427.

27 G. Cantele, Y. S. Lee, D. Ninno and N. Marzari, Nano Lett., 2009, 9, 3425-3429.

28 D. Selli, M. Baldoni, A. Sgamellotti and F. Mercuri, Nanoscale, 2012, 4, 1350.

29 J. Martin, N. Akerman, G. Ulbricht, T. Lohmann, J. H. Smet, K. von Klitzing and A. Yacoby, Nat. Phys., 2008, 4, 144-148.

30 A. K. M. Newaz, Y. S. Puzyrev, B. Wang, S. T. Pantelides and K. I. Bolotin, Nat. Commun., 2012, 3, 734.

31 K. Nakada and A. Ishii, Solid State Commun., 2011, 151, 13-16.

32 P. Marconcini, A. Cresti, F. Triozon, G. Fiori, B. Biel, Y.-M. Niquet, M. Macucci and S. Roche, ACS Nano, 2012, 6, $7942-7947$.

33 A. López-Bezanilla, F. Triozon and S. Roche, Nano Lett., 2009, 9, 2537-2541.

34 B. Biel, X. Blase, F. Triozon and S. Roche, Phys. Rev. Lett., 2009, 102, 096803.

35 L. Dong, S. Youkey, J. Bush, J. Jiao, V. M. Dubin and R. V. Chebiam, J. Appl. Phys., 2007, 101, 024320.

36 L. Yan, C. Punckt, I. A. Aksay, W. Mertin and G. Bacher, Nano Lett., 2011, 11, 3543-3549.

37 P. Willke, T. Druga, R. G. Ulbrich, M. A. Schneider and M. Wenderoth, Nat. Commun., 2015, 6, 6399.
38 J. Kautz, J. Jobst, C. Sorger, R. M. Tromp, H. B. Weber and S. J. van der Molen, Sci. Rep., 2015, 5, 13604.

39 J. P. Perdew, K. Burke and M. Ernzerhof, Phys. Rev. Lett., 1996, 77, 3865-3868.

40 J. T. Falkenberg and M. Brandbyge, Beilstein J. Nanotechnol., 2015, 6, 1603-1608.

41 D. A. Areshkin and B. K. Nikolić, Phys. Rev. B: Condens. Matter Mater. Phys., 2010, 81, 155450.

42 L. Bengtsson, Phys. Rev. B: Condens. Matter Mater. Phys., 1999, 59, 12301-12304.

43 M. Otani and O. Sugino, Phys. Rev. B: Condens. Matter Mater. Phys., 2006, 73, 115407.

44 T. Brumme, M. Calandra and F. Mauri, Phys. Rev. B: Condens. Matter Mater. Phys., 2014, 89, 1-11.

45 T. Brumme, M. Calandra and F. Mauri, Phys. Rev. B: Condens. Matter Mater. Phys., 2015, 91, 155436.

46 G. Schull, T. Frederiksen, M. Brandbyge and R. Berndt, Phys. Rev. Lett., 2009, 103, 206803.

47 T. Frederiksen, G. Foti, F. Scheurer, V. Speisser and G. Schull, Nat. Commun., 2014, 5, 3659.

48 N. L. Schneider, N. Néel, N. P. Andersen, J. T. Lü, M. Brandbyge, J. Kröger and R. Berndt, J. Phys.: Condens. Matter, 2015, 27, 015001.

49 A. Kokalj, J. Mol. Graphics Modell., 1999, 17, 176-179. 